\title{
INFINITE-DIMENSIONAL WHITEHEAD AND VIETORIS THEOREMS IN SHAPE AND PRO-HOMOTOPY
}

\author{
BY \\ DAVID A. EDWARDS AND ROSS GEOGHEGAN( ${ }^{1}$ )
}

\begin{abstract}
In Theorem 3.3 and Remark 3.4 conditions are given under which an infinite-dimensional Whitehead theorem holds in pro-homotopy. Applications to shape theory are given in Theorems 1.1, 1.2, 4.1 and 4.2.
\end{abstract}

1. Introduction. Whitehead theorems in the shape theory of finite-dimensional spaces have been proved by Moszyńska [26] and by MardeSic [22], while in [7] we proved a Whitehead theorem in pro-homotopy theory for inverse systems of complexes whose dimensions are bounded. On first sight, the prospects for removing the hypotheses of finite dimension looked bleak, because of the counterexamples in [13], [11], [1, p. 35], [5] and [4]. However, by restricting ourselves just enough to avoid these counterexamples we have been able to prove reasonable theorems. We were led to them by reading the papers of Borsuk [31] and of Kozlowski and Segal [17]. For compact metric spaces (compacta) their theorem reads: a movable compactum whose shape groups are trivial is shape equivalent to a point. Our generalizations of this are Theorems 4.1 and 4.2 below. Confining ourselves in this introduction to the compact metric case, our theorem becomes:

THEOREM 1.1. Let $\varphi: X \rightarrow Y$ be a pointed shape morphism between pointed connected compacta, which induces isomorphisms on the (inverse limit) shape groups. If $X$ is movable and $Y$ is an FANR (in the pointed sense) then $\varphi$ is a pointed shape equivalence.

This is proved by combining Theorem 4.2, below, with Theorem 5.1 of our paper [7].

Theorem 1.1 has a geometrical consequence of some interest. A map $f: X \rightarrow Y$ between compacta is called a CE map (or cell-like map or Vietoris map) if for each $y \in Y, f^{-1}(y)$ is shape equivalent to a point. If $X$ and $Y$ are ANR's then $f$ must be a homotopy equivalence (see [12], [15] and the references

Received by the editors December 16, 1974 and, in revised form, May 16, 1975.

AMS (MOS) subject classifications (1970). Primary 55D99; Secondary 14 F99.

(1) This work was done while the second-named author was visiting at the University of Georgia, whose hospitality he gratefully acknowledges. He was supported in part by N. S. F. Grant PO 38761. 
therein). If $X$ and $Y$ are finite-dimensional compacta, $f$ must be a shape equivalence (see [28] and [15]): Anderson (unpublished) was able to remove the requirement that $X$ be finite dimensional. But if one also removes the requirement that $Y$ be finite dimensional, counterexamples exist: Taylor [29] constructed a CE map from a nonmovable compactum onto the Hilbert Cube, while Keesling [14] modified this example to get a CE map from the Hilbert Cube onto a nonmovable compactum: clearly these cannot be shape equivalences. Kozlowski and Segal have gone further, by constructing [32] a CE map between movable compacta of different shapes. The theorems in this paper imply the following "infinite-dimensional Vietoris theorem" (which is proved by combining Theorem 1.1, above, with Theorem 2.3 of K. Kuperberg's paper [18]).

THEOREM 1.2. Let $f:(X, x) \rightarrow(Y, y)$ be a $C E$ map between pointed connected compacta. If $(X, x)$ is movable and $(Y, y)$ is an FANR (in the pointed sense), then $f$ is a pointed shape equivalence.

Our principal tool is a Whitehead theorem in pro-homotopy, Theorem 3.3. Roughly, it says that a weak equivalence in pro-homotopy from an inverse system $\left\{X_{\alpha}\right\}$ of finite-dimensional complexes to a finite-dimensional complex $Y$ is an equivalence provided $\left\{X_{\alpha}\right\}$ is movable. The point is that the dimensions of the complexes $X_{\alpha}$ need not be bounded.

In Remarks 3.4 and 4.4 we indicate how the hypotheses on $X$ and $Y$ of Theorem 1.1 can be replaced by the hypothesis that $\varphi$ be a "movable morphism."

Note ADDED MAY 1, 1975. J. Dydak [39] has extended our shape theoretic results. It is not clear whether his methods can be adapted to improve our pro-homotopy results.

2. Notation, terminology and a lemma. We follow the notational conventions set out in $\S \S 2$ and 3 of [7]. The principal items are listed below. Shape terminology is introduced in $\$ 4$.

If $C$ is a category, pro- $C$ denotes a certain category of inverse systems in $C$ indexed by directed sets: for a description of the morphisms and other properties of pro-C see [5] or [22]; for the original more general version see the Appendix of [1]. $C_{\text {maps }}$ denotes the category whose objects are the morphisms of $C$ and whose morphisms from an object $f$ to an object $g$ are the commutative square diagrams

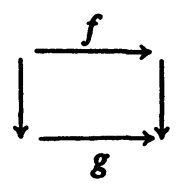

in $C$. There is an obvious functor pro- $\left(C_{\text {maps }}\right) \rightarrow(\text { pro-C })_{\text {maps }}$ and we say that the object $\left\{X_{\alpha} \stackrel{f_{\alpha}}{\longrightarrow} Y_{\alpha}\right\}$ of pro-( $\left(C_{\text {maps }}\right)$ "induces" its image $\left\{X_{\alpha}\right\} \stackrel{\left\{f_{\alpha}\right\}}{\longrightarrow}\left\{Y_{\alpha}\right\}$ under this functor: see $\S 3$ of [7]. 
We suppress bonding morphisms and the indexing directed set, denoting an object of pro-C by $\left\{X_{\alpha}\right\} .\left\{X_{\alpha}\right\}$ is movable if for each $\alpha$ there exists $\beta \geqslant \alpha$ such that for all $\gamma \geqslant \beta$ the bond $p_{\alpha \beta}: X_{\beta} \rightarrow X_{\alpha}$ factors as $p_{\alpha \beta}=p_{\alpha \gamma} \circ r^{\beta \gamma}$ where $r^{\beta \gamma}: X_{\beta} \rightarrow X_{\gamma}$ is a morphism of $C .\left\{X_{\alpha}\right\}$ is uniformly movable if for each $\alpha$ there exists $\beta \geqslant \alpha$ such that the bond $p_{\alpha \beta}$ factors as $p_{\alpha \beta}=p_{\alpha} \circ r^{\beta}$ where $r^{\beta}: X_{\beta}$ $\rightarrow\left\{X_{\alpha}\right\}$ is a morphism of pro-C and $p_{\alpha}:\left\{X_{\alpha}\right\} \rightarrow X_{\alpha}$ is the projection morphism of pro-C. ( $C$ is, of course, embedded as a full subcategory of pro- $C$.)

A directed set is closure finite if each element has only finitely many predecessors.

Categories used include: Groups (groups and homorphisms); $T_{0}$ (pointed connected topological spaces and pointed continuous functions); $H T_{0}$ (the homotopy category corresponding to $T_{0}$ ); $C W_{0}$ (pointed connected $\mathrm{CW}$ complexes and pointed continuous functions); $H_{0}$ (the homotopy category corresponding to $C W_{0}$ ); $H T_{0 \text {,pairs }}$ (pointed pairs of connected spaces and pointed homotopy classes of maps); $H_{0, p a i r s}$ (pointed pairs of connected $\mathrm{CW}$ complexes and pointed homotopy classes of maps).

We call an object of pro-Groups a pro-group. We always suppress base points of spaces.

The definition of uniform movability becomes simpler in the case of progroups. A pro-group $G \equiv\left\{G_{\alpha}\right\}$ is (clearly) uniformly movable if and only if for each $\alpha$ there exists $\beta \geqslant \alpha$ such that the bond $p_{\alpha \beta}: G_{\beta} \rightarrow G_{\alpha}$ factors as $p_{\alpha \beta}=$ $p_{\alpha} \circ r^{\beta}$ where $r^{\beta}: G_{\beta} \rightarrow \lim G$ is a homomorphism and $p_{\alpha}: \lim G \rightarrow G_{\alpha}$ is projection on the $\alpha$ factor.

LEMMA 2.1. Let $G \equiv\left\{G_{\alpha}\right\}$ be a uniformly movable pro-group. Let $H$ be a group, let $f: G \rightarrow H$ be a morphism of pro-Groups and let $p: \lim G \rightarrow G$ be the projection morphism. If $\widetilde{f} \equiv f \circ p$ is an isomorphism (of groups) then $f$ is an isomorphism (of pro-groups).

Proof. The required inverse to $f$ is $p \circ \widetilde{f}^{-1}$. It is trivial that $f \circ\left(p \circ \widetilde{f}^{-1}\right)$ is the identity of $G$. To show that $\left(p \circ \tilde{f}^{-1}\right) \circ f$ is the identity of $\left\{G_{\alpha}\right\}$ it is enough to show that given $\alpha$ there exists $\gamma \geqslant \alpha$ such that $p_{\alpha} \circ \tilde{f}^{-1} \circ f_{\beta} \circ p_{\beta \gamma}=$ $p_{\alpha \gamma}$. Since $G$ is uniformly movable the above remark implies that there exist $\beta \geqslant \alpha$ and $r^{\beta}: G_{\beta} \rightarrow \lim G$ such that $p_{\alpha \beta}=p_{\alpha} \circ r^{\beta}$. Let $f$ be represented by homomorphisms $f_{\alpha}: G_{\alpha} \rightarrow H$. Choose $\gamma \geqslant \beta$ such that $f_{\beta} \circ p_{\beta \gamma}=f_{\alpha} \circ p_{\alpha \beta} \circ$ $p_{\beta \gamma}$. Then

So

$$
f_{\beta} \circ p_{\beta \gamma}=f_{\alpha} \circ p_{\alpha} \circ r^{\beta} \circ p_{\beta \gamma}=\tilde{f} \circ r^{\beta} \circ p_{\beta \gamma}
$$

$$
p_{\alpha} \circ \tilde{f}^{-1} \circ f_{\beta} \circ p_{\beta \gamma}=p_{\alpha} \circ r^{\beta} \circ p_{\beta \gamma}=p_{\alpha \beta} \circ p_{\beta \gamma}=p_{\alpha \gamma}
$$


3. A Whitehead theorem in pro-homotopy. The principal result here is Theorem 3.3. Lemma 3.1 and Proposition 3.2 are the modifications needed to obtain an infinite-dimensional Whitehead theorem from [22] and [7].

If $f: X \rightarrow Y$ is a morphism of $T_{0}, M(f)$ denotes the reduced mapping cylinder of $f$, and $X$ is regarded as a subset of $M(f)$ in the usual manner. Thus $(M(f), X)$ is an object of $T_{0, \text { pairs. }}$. If $f \equiv\left\{X_{\alpha} \stackrel{f_{\alpha}}{\longrightarrow} Y_{\alpha}\right\}$ is an object of pro$\left(T_{0, \text { maps }}\right)$ then $M(f) \equiv\left\{\left(M\left(f_{\alpha}\right), X_{\alpha}\right)\right\}$ is a well-defined object of pro-( $\left.T_{0, \text { airs }}\right)$ and so induces an object of pro-(HT $(H$,pairs $)$; see $\S 3$ of [7].

LEMMA 3.1. Let $f \equiv\left\{X_{\alpha} \stackrel{f_{\alpha}}{\longrightarrow} Y_{\alpha}\right\}$ be an object of pro-(CW, $\left(C W_{0, \text { aps }}\right)$ whose domain $\left\{X_{\alpha}\right\}$ is movable in pro- $H_{0}$ and whose range $\left\{Y_{\alpha}\right\}$ is such that every $Y_{\alpha}$ is the same (pointed) complex $Y$, and every bond $Y_{\beta} \rightarrow Y_{\alpha}$ is the identity map. Then $\left\{\left(M\left(f_{\alpha}\right), X_{\alpha}\right)\right\}$ is movable in pro-(HT 0 ,pairs $)$.

PROOF. Let $p_{\alpha \beta}: X_{\beta} \rightarrow X_{\alpha}$ denote the appropriate bond of $\left\{X_{\alpha}\right\}$. Given $\alpha$, there exists $\beta \geqslant \alpha$ such that for all $\gamma \geqslant \beta$ there is a pointed map $r^{\beta \gamma}: X_{\beta} \rightarrow$ $X_{\gamma}$ with the property that $p_{\alpha \beta}$ is pointedly homotopic to $p_{\alpha \gamma} \circ r^{\beta \gamma}$. By Theorem 2.8.9 of [37], $p_{\alpha \gamma}$ may be replaced by a fibration: to be precise, there exist a pointed fibration $p_{\alpha \gamma}^{\prime}: X_{\gamma}^{\prime} \rightarrow X_{\alpha}$, and an inclusion $i: X_{\gamma} \rightarrow X_{\gamma}^{\prime}$ as a pointed strong deformation retract, such that $p_{\alpha \gamma}^{\prime} \circ i=p_{\alpha \gamma}$. Since $p_{\alpha \gamma}^{\prime}$ is a fibration, there is a pointed map $s^{\beta \gamma}: X_{\beta} \rightarrow X_{\gamma}^{\prime}$ such that $p_{\alpha \gamma}^{\prime} \circ s^{\beta \gamma}=p_{\alpha \beta}$. Letting $f_{\gamma}^{\prime}=$ $f_{\alpha} \circ p_{\alpha \gamma}^{\prime}$ we have a commutative diagram in $C W_{0}$ :

(*)

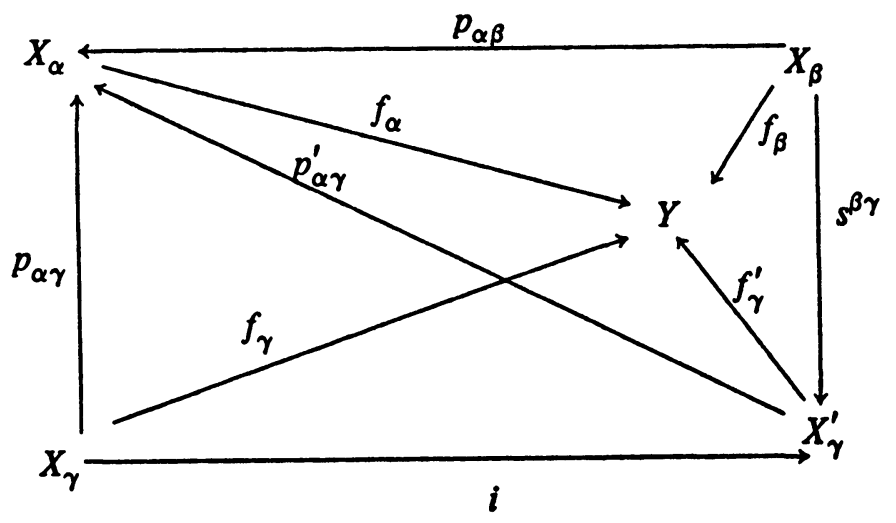

From it, we obtain a commutative diagram in $T_{0, p a i r s}$ :

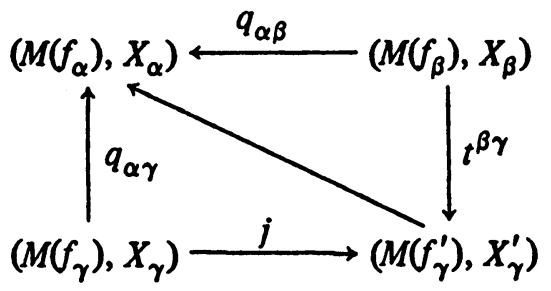


$j$ induces pointed homotopy equivalences $M\left(f_{\gamma}\right) \rightarrow M\left(f_{\gamma}^{\prime}\right)$ and $X_{\gamma} \rightarrow X_{\gamma}^{\prime}$; by Lemma 1 of [34], we could deduce that $j$ induces an isomorphism in $H T_{0 \text {,pairs }}$ if we knew that $\left(M\left(f_{\gamma}\right), X_{\gamma}\right)$ and $\left(M\left(f_{\gamma}^{\prime}\right), X_{\gamma}^{\prime}\right)$ were isomorphic in $H T_{0 \text {,pairs }}$ to pointed CW pairs. It is not hard to show that $\left(M\left(f_{\gamma}\right), X_{\gamma}\right)$ has this property $\left(f_{\gamma}\right.$ is homotopic to a cellular map; use Lemma 3.9 of [7]). But it is not clear that the same is true of $\left(M\left(f_{\gamma}^{\prime}\right), X_{\gamma}^{\prime}\right)$. To avoid the problem, we apply the composite functor "geometric realization of the singular complex" [33], $|\cdot| \circ S: T_{0} \rightarrow$ $C W_{0}$, to the diagram (*), and thus obtain the following commutative diagram in $C W_{0, \text { pairs }}$, analogous to $(* *)$ :

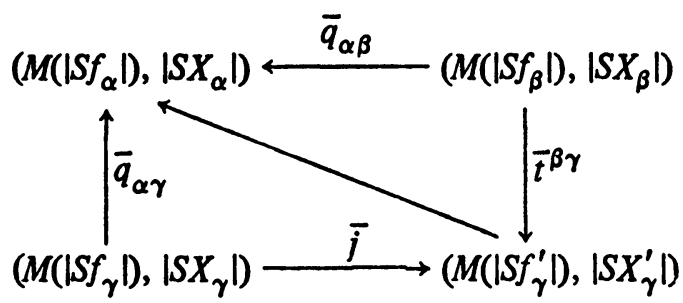

where the maps are obtained from those of $(* *)$ in the obvious way. Now, Lemma 1 of [34] implies that $\bar{j}$ induces an isomorphism in $H T_{0, p a i r s}$. It follows that $\bar{q}_{\alpha \beta}$ can be lifted in $H T_{0 \text {,pairs }}$ through $\bar{q}_{\alpha \gamma}$, so that $\left\{\left(M\left(\left|S f_{\alpha}\right|\right),\left|S X_{\alpha}\right|\right)\right\}$ is movable in $H T_{0, p a i r s}$ (where the bonds are induced by the maps $\bar{q}_{\alpha \beta}$ ). The argument is completed by observing that $\left\{\left(M\left(\left|S f_{\alpha}\right|\right),\left|S X_{\alpha}\right|\right)\right\}$ is isomorphic in pro$H T_{0, \text { pairs }}$ to $\left\{\left(M\left(f_{\alpha}\right), X_{\alpha}\right)\right\}$. To see this, observe that there is a commutative diagram in $T_{0, \text { pairs }}$

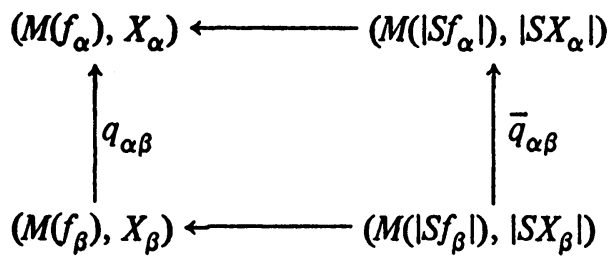

whose horizontal morphisms are induced by the canonical maps $\left|S X_{\alpha}\right| \rightarrow X_{\alpha}$, $|S Y| \rightarrow Y$, etc. As explained above $\left(M\left(f_{\alpha}\right), X_{\alpha}\right)$ is isomorphic in $H T_{0, \text { pairs }}$ to a pointed $\mathrm{CW}$ pair; and $\left(M\left(\left|S f_{\alpha}\right|\right),\left|S X_{\alpha}\right|\right)$ is itself a pointed $\mathrm{CW}$ pair. So, by Lemma 1 of [34], the horizontal morphisms are isomorphisms in $H T_{0, \text { pairs }}$. Since $\left\{M\left(f_{\alpha}\right)\right\}$ is isomorphic to a movable object, it is itself movable.

Proposition 3.2. Let $\left\{\left(P_{\alpha}, P_{\alpha}^{\prime}\right)\right\}$ be a movable object of pro-( $\left.H_{0, \text { pairs }}\right)$ indexed by a closure finite directed set. Assume that each $P_{\alpha}$ is a finite-dimensional simplicial complex and that $P_{\alpha}^{\prime}$ is a subcomplex of $P_{\alpha^{*}}$ If $\left\{\pi_{k}\left(P_{\alpha}, P_{\alpha}^{\prime}\right)\right\}$ is trivial for all $k$, then the "inclusion" $\left\{P_{\alpha}^{\prime}\right\} \rightarrow\left\{P_{\alpha}\right\}$ is an isomorphism in pro- $H_{0}$. 
Proof. The proof is almost identical to that of Theorem 2 of [22]. Movability makes unnecessary the hypothesis in [22] that the dimensions of the complexes $P_{\alpha}$ be bounded. For each $\alpha$ choose $\beta(\alpha) \geqslant \alpha$ such that for every $\gamma \geqslant$ $\beta(\alpha)$ there exists a morphism of $H_{0, \text { pairs }}, s^{\beta \gamma}:\left(P_{\beta}, P_{\beta}^{\prime}\right) \rightarrow\left(P_{\gamma}, P_{\gamma}^{\prime}\right)$, such that $q_{\alpha \gamma} \circ s^{\beta \gamma}=q_{\alpha \beta}$ where $q_{\lambda \mu}:\left(P_{\lambda}, P_{\lambda}^{\prime}\right) \rightarrow\left(P_{\mu}, P_{\mu}^{\prime}\right)$ denotes the appropriate bonding morphism. Following 2.3 of [22], assume $\beta(\alpha) \leqslant \beta(\bar{\alpha})$ whenever $\alpha \leqslant \bar{\alpha}$.

Claim 1. For each $\alpha$, each pointed pair of finite-dimensional simplicial complexes $\left(K, K^{\prime}\right)$ and each pointed map $\varphi:\left(K, K^{\prime}\right) \rightarrow\left(P_{\beta(\alpha)}, P_{\beta(\alpha)}^{\prime}\right)$ there exists a pointed map $\psi: K \rightarrow P_{\alpha}^{\prime}$ such that (inclusion) $\circ \psi$ is pointedly homotopic to (bond) $\circ \varphi$ in $P_{\alpha}$ and $\psi \mid K^{\prime}$ is pointedly homotopic to (bond) $\circ \varphi \mid K^{\prime}$ in $P_{\alpha}^{\prime}$.

Proof of Claim 1. Apply Lemma $1(\S 6.2)$ of [22] with $n+1=\operatorname{dim} K$ and $\alpha^{*} \geqslant \beta(\alpha)$ : movability implies that $\varphi$ can be lifted to $\left(P_{\alpha^{*}}, P_{\alpha^{*}}^{\prime}\right)$, hence $\psi$ exists.

Claim 2. Given $\alpha$ and a pointed finite-dimensional complex $L$, let $\varphi_{0}, \varphi_{1}$ : $L \rightarrow P_{\beta(\alpha)}^{\prime}$ be pointed maps such that (inclusion) $\circ \varphi_{0}$ and (inclusion) $\circ \varphi_{1}$ are pointedly homotopic in $P_{\beta(\alpha)}$. Then (bond) $\circ \varphi_{0}$ and (bond) $\circ \varphi_{1}$ are pointedly homotopic in $P_{\alpha}^{\prime}$.

Proof of Claim 2. Apply Lemma 2 (§6.3) of [22] with $n=\operatorname{dim} L$ : movability implies that $\varphi_{0}$ and $\varphi_{1}$ can be lifted to $P_{\alpha^{*}}^{\prime}$, and the claim follows.

The remainder of the proof is similar to the corresponding proof in $\$ 6.4$ of [22]. Claims 1 and 2 are used in place of Lemmas 1 and 2 of [22].

THEOREM 3.3. Let $Y$ be a pointed complex, $\left\{X_{\alpha}\right\}$ an object of pro-CW and $g: X \rightarrow Y$ a morphism of pro- $H_{0}$. Assume $Y$ and each $X_{\alpha}$ are finite dimensional, and that the object of pro- $H_{0}$ induced by $\left\{X_{\alpha}\right\}$ is movable. If $g_{\#}$ : $\left\{\pi_{k}\left(X_{\alpha}\right)\right\} \rightarrow \pi_{k}(Y)$ is an isomorphism (in the category pro-Groups) for every $k$, then $g$ induces an isomorphism of pro- $H_{0}$.

Proof. The proof is similar to that of Theorem 3.1 of [7]. Since $Y$ is a complex, we may represent $g$ by a morphism of pro- $C W_{0}$ and hence replace it by an object $f \equiv\left\{X_{\gamma}^{\prime} \stackrel{f_{\gamma}}{\longrightarrow} Y_{\gamma}^{\prime}\right\}$ of pro-( $\left(C W_{0, \text { maps }}\right)$ indexed by a closure finite directed set such that: $\left\{X_{\gamma}^{\prime}\right\}$ is movable, each $X_{\gamma}^{\prime}$ is finite dimensional, each $Y_{\gamma}^{\prime}$ is $Y$, and each bond of $\left\{Y_{\gamma}^{\prime}\right\}$ is the identity map; see $\S 3$ of [7]. $f_{\#}:\left\{\pi_{k}\left(X_{\gamma}^{\prime}\right)\right\}$ $\rightarrow\left\{\pi_{k}\left(Y_{\gamma}^{\prime}\right)\right\}$ is an isomorphism of pro-groups for each $k$. By Lemma 3.8 of [7], $\left\{\pi_{k}\left(M\left(f_{\gamma}\right), X_{\gamma}^{\prime}\right)\right\}$ is trivial, where $\left\{M\left(f_{\gamma}\right)\right\}$ is the reduced mapping cylinder object of pro- $C W_{0}$ corresponding to $f$ (see $\S 3$ of [7]). Each $M\left(f_{\gamma}\right)$ is finite dimensional. By Lemma 3.1, above, $\left\{\left(M\left(f_{\gamma}\right), X_{\gamma}^{\prime}\right)\right\}$ is movable in pro-(HT ,pairs $)$. The rest of the proof is as in [7], except that Proposition 3.12 of [7] is replaced by the above Proposition 3.2.

REMARK 3.4. There is a variation on Theorem 3.3. Following [9], define $H-C W_{0, \text { maps }}$ to be the category whose objects are those of $C W_{0, \text { maps }}$ and whose 
morphisms are homotopy classes of morphisms of $C W_{0, \text { maps }}$, where two morphisms $\left(a_{1}, a_{2}\right)$ and $\left(b_{1}, b_{2}\right)$ from $f: X \rightarrow Y$ to $f^{\prime}: X^{\prime} \rightarrow Y^{\prime}$ are defined to be homotopic if there is a morphism $\left(\theta_{1}, \theta_{2}\right)$ from $f \times 1: X \times I \rightarrow Y \times I$ to $f^{\prime}: X^{\prime} \rightarrow Y^{\prime}$ such that $\theta_{i}$ is a homotopy between $a_{i}$ and $b_{i}, i=1,2$. Call an object $\left\{X_{\gamma}^{\prime} \stackrel{f_{\gamma}}{\longrightarrow} Y_{\gamma}^{\prime}\right\}$ of pro-CW, maps $H$-movable if it induces a movable object of pro-(H-CW $\left.W_{0, \text { aps }}\right)$. Call a morphism $g:\left\{X_{\alpha}\right\} \rightarrow\left\{Y_{\beta}\right\}$ of pro-CW movable if $g$ is isomorphic in (pro- $\left.H_{0}\right)_{\text {maps }}$ to the object of (pro- $\left.H_{0}\right)_{\text {maps }}$ induced by such an $H$-movable $\left\{f_{\gamma}\right\}$. If each $X_{\alpha}$ and each $Y_{\beta}$ is finite dimensional, if $g$ is movable, and if $g$ induces isomorphisms of homotopy pro-groups, then $g$ induces an isomorphism in pro- $H_{0}$. The proof is similar to that of Theorem 3.3. The hypotheses make it possible to by-pass Lemma 3.1: clearly $\left\{\left(M\left(f_{\gamma}\right), X_{\gamma}^{\prime}\right)\right\}$ is movable in pro-( $\left.H T_{0, \text { pairs }}\right)$.

4. Whitehead theorems in shape. All spaces mentioned will be paracompact Hausdorff, so our shape theory may be understood either in the sense of [21] or [27], since these two theories agree on such spaces [19], [25]. For compact Hausdorff spaces these theories agree with that of [23], and for compact metric spaces they agree with that of [3] (see [24]).

We refer the reader to [25] or to $\S 3$ of [22] for an account of how the shape theory of spaces is fully and faithfully reflected in pro-homotopy theory. In particular, if $X$ and $Y$ are pointed connected spaces, there is a functorial bijection between the (pointed) shape morphisms from $X$ to $Y$ and the morphisms of pro- $H_{0}$ from $\left\{X_{\alpha}\right\}$ to $\left\{Y_{\beta}\right\}$, where $\left\{X_{\alpha}\right\}$ and $\left\{Y_{\beta}\right\}$ are objects of pro- $H_{0}$ (unique up to isomorphism) which are "associated" with $X$ and $Y$ respectively. A shape morphism $\varphi: X \rightarrow Y$ is a weak shape equivalence if the corresponding $f:\left\{X_{\alpha}\right\} \rightarrow\left\{Y_{\beta}\right\}$ induces isomorphisms $f_{\#}:\left\{\pi_{k}\left(X_{\alpha}\right)\right\} \rightarrow\left\{\pi_{k}\left(Y_{\beta}\right)\right\}$ in pro-Groups for each $k \geqslant 1$. $\varphi$ is a very weak shape equivalence if

$$
f_{\#}: \underset{\alpha}{\lim }\left\{\pi_{k}\left(X_{\alpha}\right)\right\} \rightarrow \underset{\beta}{\lim }\left\{\pi_{k}\left(Y_{\beta}\right)\right\}
$$

is an isomorphism in Groups for each $k \geqslant 1 . X$ is movable [resp. uniformly movable] if $\left\{X_{\alpha}\right\}$ is movable [resp. uniformly movable] in pro- $H_{0}$.

Every object of pro- $C W_{0}$ gives rise to an object of pro- $H_{0}$, but (apart from the case of countably indexed systems) it is unknown whether every object of pro- $H_{0}$ "comes from" an object of pro- $C W_{0}$. The Vietoris functor [27] allows one to associate objects "coming from" pro- $C W_{0}$ with spaces, but the complexes involved are infinite dimensional. It is for these reasons that we confine ourselves to compact Hausdorff spaces in the theorems which follow.

THEOREM 4.1. Let $X$ be a movable pointed connected compact Hausdorff space, let $Y$ be pointed shape equivalent to a pointed connected CW complex 
and let $\varphi: X \rightarrow Y$ be a pointed shape morphism. If $\varphi$ is a weak shape equivalence, it is a pointed shape equivalence.

Proof. Assume $Y$ is a $\mathrm{CW}$ complex. First assume $Y$ is a finite-dimensional complex. As we shall see, no generality is lost by this.

Let $\left\{X_{\alpha}\right\}$ be an object of pro-CW $W_{0}$ whose inverse limit is homeomorphic to $X$. Then $\left\{X_{\alpha}\right\}$ is associated with $X$ in the sense of [25]. Let $g:\left\{X_{\alpha}\right\} \rightarrow Y$ be a morphism of pro- $H_{0}$ associated with $\varphi$ in the sense of [25]. By Theorem $3.3, g$ induces an isomorphism in pro- $H_{0}$. Hence, by [25], $\varphi$ is a shape equivalence.

If $Y$ is not finite dimensional we show that it must be (pointed) homotopy equivalent to a finite-dimensional complex. Since $X$ is compact, $g$ may be represented by a continuous map $g_{\alpha_{0}}: X_{a_{0}} \rightarrow Y$ for some $\alpha_{0}$, and hence $g$ factors through a finite subcomplex $K$ of $Y$. So $\tilde{g}:\left\{\tilde{X}_{\alpha}\right\} \rightarrow \widetilde{Y}$ factors through $\widetilde{K}$ (where we have applied the pointed universal cover functor $\sim$ ). Since $g$ is a weak equivalence in pro- $H_{0}$, so also is $\tilde{g}$. Hence $g$ and $\tilde{g}$ are -isomorphisms $[1, \S 4]$; therefore, they induce isomorphisms on homology pro-groups and cohomology groups with every possible coefficient bundle (see 4.4 of [1]). Since $K$ and $\widetilde{K}$ are finite dimensional, the homology of $\tilde{Y}$ and the cohomology of $Y$ vanish above the dimension of $K$. By Theorem $\mathrm{E}$ of [30], $Y$ is homotopy equivalent (hence pointed homotopy equivalent) to a finite-dimensional complex.

THEOREM 4.2. Let $X$ be a uniformly movable pointed connected compact Hausdorff space, let $Y$ be pointed shape equivalent to a pointed $C W$ complex, and let $\varphi: X \rightarrow Y$ be a morphism in pointed shape. If $\varphi$ is a very weak shape equivalence, it is a pointed shape equivalence. Furthermore, if $X$ is metrizable it is only necessary to assume that $X$ is movable.

Proof. By Lemma 2.1, $\varphi$ is a weak shape equivalence, so the conclusion follows from Theorem 4.1. For metric compacta the concepts of "movable" and "uniformly movable" coincide, by [38] (see also Theorem 4.7 of [16] and Remark 6.7 of [35]) so the last statement is justified.

REMARK 4.3. Various criteria are available for deciding if a given space $Y$ is shape equivalent to a CW complex (as required in Theorems 4.1 and 4.2). See $[10],[6],[7],[8]$.

REMARK 4.4. Following Remark 3.4, one may define the notion of "movable shape morphism": the special case of "movable map" is discussed in [9]. One may then prove that if $\varphi: X \rightarrow Y$ is a movable pointed shape morphism between metric compacta and if $\varphi$ is a very weak shape equivalence, then $\varphi$ is a shape equivalence. A remark on p. 4 of [2] (incorrect as stated, but correct in the countable case) is used instead of Lemma 2.1 to show that $\varphi$ is a weak shape 
equivalence. Then Remark 3.4 is used instead of Theorem 3.3 to complete the proof. Compare with [36].

REMARK 4.5. If one interchanges the propertics of $X$ and $Y$ in Theorems 4.1 and 4.2, making $Y$ movable (or uniformly movable) and $X$ shape equivalent to a complex, the resulting "theorems" are false. Counterexamples are given in [5]. However, if one also requires $X$ to be compact metric (or, equivalently, to be an FANR: see [6]) we do not know a counterexample. Added in proof: there is none; see [39].

\section{REFERENCES}

1. M. Artin and B. Mazur, Etale homotopy, Lecture Notes in Math., vol. 100, Springer-Verlag, Berlin and New York, 1969. MR 39 \#6883.

2. M. F. Atiyah and G. B. Segal, Equivariant $K$-theory and completion, J. Differential Geometry 3 (1969), 1-18. MR 41 \#4575.

3. K. Borsuk, Concerming homotopy properties of compacta, Fund. Math. 62 (1968), 223-254. MR 37 \#811.

4. J. Draper and J. Keesling, An example concerning the Whitehead theorem in shape theory, Fund. Math. (to appear).

5. D. A. Edwards and R. Geoghegan, Compacta weak shape equivalent to ANR's, Fund. Math. 90 (1975).

6. Shapes of complexes, ends of manifolds, homotopy limits and the Wall obstruction, Ann. of Math. 101 (1975), 521-535.

7. The stability problem in shape and a Whitehead theorem in pro-homotopy, Trans. Amer. Math. Soc. 214 (1975), 261-277. (to appear).

8. - Stability theorems in shape and pro-homotopy, Trans. Amer. Math. Soc.

9. D. A. Edwards and P. T. McAuley, The shape of a map, Fund. Math. (to appear).

10. R. Geoghegan and R. C. Lacher, Compacta with the shape of finite complexes, Fund. Math. (to appear).

11. D. Handel and J. Segal, An acyclic continuum with non-movable suspensions, Bull. Acad. Polon. Sci. Sér. Sci. Math. Astronom. Phys. 21 (1973), 171-172. MR 47 \#5813.

12. W. E. Haver, Mappings between $A N R$ 's that are fine homotopy equivalences (mimeographed).

13. D. S. Kahn, An example in Cech cohomology, Proc. Amer. Math. Soc. 16 (1965), 584. MR $31 \# 4027$.

14. J. Keesling, A trivial-shape decomposition of the Hilbert cube such that the decomposition space is not an absolute retract, Bull. Acad. Polon. Sci. Sér. Sci. Math. Astronom. Phys. (to appear).

15. G. Kozlowski, Images of ANR's (preprint).

16. G. Kozlowski and J. Segal, Locally well-behaved paracompacta in shape theory, Fund. Math. (to appear).

17. - Movability and shape connectivity, Fund. Math. (to appear).

18. K. Kuperberg, Two Vietoris-type isomorphism theorems in Borsuk's theory of shape, concerning the Vietoris-Cech homology and Borsuk's fundamental groups, Studies in Topology, Academic Press, New York, 1975.

19. J. Levan, Shape theory, Doctoral Dissertation, University of Kentucky, 1973.

20. A. T. Lundell and S. Weingram, The topology of CW complexes, Van Nostrand Reinhold, New York, 1969. 265-282.

21. S. Mardełić, Shapes for topological spaces, General Topology and Appl. 3 (1973),

22. - On the Whitehead theorem in shape theory. I, Fund. Math. (to appear). 
23. S. Mardesić and J. Segal, Shapes of compacta and ANR-systems, Fund. Math. 72 (1971), 41-59. MR 45 \#7686.

24. Equivalence of the Borsuk and the ANR-system approach to shapes, Fund. Math. 72 (1971), 61-68. MR 46 \#850.

25. K. Morita, On shapes of topological spaces, Fund. Math. 86 (1975), 251-259.

26. M. Moszynska, The Whitehead theorem in the theory of shape, Fund. Math. 80 (1973), $221-263$.

27. T. Porter, Cech homotopy. I, II, J. London Math. Soc. (2) 6 (1973), 429-436, 667-675. MR 47 \#9613, 50 \#8517a.

28. R. B. Sher, Realizing cell-like maps in euclidean space, General Topology and Appl. 2 (1972), 75-89. MR 46 \#2683.

29. J. L. Taylor, A counter-example in shape theory, Bull. Amer. Math. Soc. 81 (1975), 629-632.

30. C. T. C. Wall, Finiteness conditions for CW-complexes, Ann. of Math. (2) 81 (1965), 56-69. MR 30 \#1515.

31. K. Borsuk, Some remarks on shape properties of compacta, Fund. Math. 85 (1974), 185-195.

32. G. Kozlowski and J. Segal, Local behavior and the Vietoris and Whitehead theorems in shape theory, Fund. Math. (to appear).

33. J. P. May, Simplicial objects in algebraic topology, Van Nostrand Math. Studies, no. 11, Van Nostrand, Princeton, N. J., 1967. MR 36 \#5942.

34. J. W. Milnor, On spaces having the homotopy type of $\mathrm{CW}$-complex, Trans. Amer. Math. Soc. 90 (1959), 272-280. MR 20 \#6700.

35. M. Moszyńska, Uniformly movable compact spaces and their algebraic properties, Fund. Math. 77 (1972), 125-144. MR 48 \#1224.

36. Concerning the Whitehead theorem for movable compacta, Fund. Math. (to appear).

37. E. H. Spanier, Algebraic topology, McGraw-Hill, New York, 1966. MR 35 \#1007.

38. S. Spiesź, Movability and uniform movability, Bull. Acad. Polon. Sci. Sér. Sci. Math. Astronom. Phys. 22 (1974), 43-45.

39. J. Dydak, Some remarks concerning the Whitehead theorem in shape theory, Bull. Acad. Polon. Sci. Sér. Sci. Math. Astronom. Phys. 23 (1975), 437-445.

DEPARTMENT OF MATHEMATICS, STATE UNIVERSITY OF NEW YORK, BINGHAMTON, NEW YORK 13901 